\section{What is mumps?}

Mumps is an acute disease caused by the mumps virus. In the past, mumps infection was very common in childhood. Due to immunisation, it has become less common in Australia.

\section{What are the symptoms?}

- Common symptoms of mumps are fever, loss of appetite, tiredness and headache, followed by swelling and tenderness of the salivary glands. One or both of the parotid salivary glands are frequently affected (the parotid glands are salivary glands in the bottom of the cheeks near the angle of the jaw). Sometimes other salivary glands are also affected.

- About one third of people who have mumps infection do not show any symptoms at all.

- Mumps is usually a more severe illness in people who become infected after puberty.

- Complications from the mumps are rare but can include inflammation of the brain (encephalitis), the lining of the brain and spinal cord (meningitis), testicles (orchitis), ovaries (oophoritis) and pancreas (pancreatitis). Mumps can also cause spontaneous abortion and hearing loss. Sterility (inability to have children) in males is an extremely rare complication.

\section{How is it spread?}

- Mumps is spread when a person breathes in the mumps virus that has been coughed or sneezed into the air by an infectious person. The mumps virus can also spread from person to person via direct contact with infected saliva.

- People with mumps can be infectious up to seven days before and nine days after swelling of the salivary glands begins. Maximum infectiousness occurs between two days before to four days after the onset of symptoms.

- The time from being exposed to the virus and becoming sick can range from 12 to 25 days but is most commonly 16 to 18 days.

\section{Who is at risk?}

Anyone who comes into contact with infectious mumps can get mumps, unless they have been infected in the past, or have been immunised with MMR vaccine.

\section{How is it prevented?}

- People with mumps should stay at home until the swelling in the salivary glands disappears, or up to nine days after the onset of swelling (whichever is sooner) to help stop spreading the virus to others.

- A vaccine that only protects against mumps is not available in Australia; however, MMR vaccine protects against mumps, measles and rubella and is part of the standard vaccination schedule. MMR vaccine should be given to children at age 12 months and again at four years.

- People born after 1965 should ensure they have received two doses of MMR vaccine.

\section{How is it diagnosed?}

The doctor may diagnose mumps based on the person's symptoms and signs alone. A blood test or sample from the throat, urine or spinal cord fluid can confirm the diagnosis.

\section{How is it treated?}

There is no specific treatment for mumps. Simple analgesics may reduce pain and fever. Warm or cold packs to the swollen glands may provide relief.

\section{What is the public health response?}

- Laboratories, school principals and directors of childcare centres must report all cases of mumps to their local public health unit. These reports provide statistics to help understand trends in the incidence of mumps in the community.

- People with mumps should stay away from childcare, school or work for nine days after the onset of the swelling.

\section{NSW POHEALTH}

